\title{
Resistome and Virulome of Multi-Drug Resistant E. coli ST131 Isolated from Residents of Long-Term Care Facilities in the Northern Italian Region
}

Sabrina Cherubini ${ }^{1}$, Mariagrazia Perilli ${ }^{1}+{ }^{\mathbb{D}}$, Anna Maria Azzini ${ }^{2}$, Evelina Tacconelli ${ }^{2}$, Laura Maccacaro ${ }^{3}$, Alda Bazaj ${ }^{3}$, Laura Naso ${ }^{3}$, Gianfranco Amicosante ${ }^{1}$, LTCF-Veneto Working Group $¥$, Giuliana Lo Cascio ${ }^{3,4,+}$ (D) and Alessandra Piccirilli ${ }^{1, *}$

check for updates

Citation: Cherubini, S.; Perilli, M.; Azzini, A.M.; Tacconelli, E.; Maccacaro, L.; Bazaj, A.; Naso, L.; Amicosante, G.; LTCF-Veneto Working Group; Lo Cascio, G.; et al. Resistome and Virulome of MultiDrug Resistant E. coli ST131 Isolated from Residents of Long-Term Care Facilities in the Northern Italian Region. Diagnostics 2022, 12, 213. https://doi.org/10.3390/ diagnostics12010213

Academic Editor: Georgina Tzanakaki

Received: 16 December 2021

Accepted: 12 January 2022

Published: 16 January 2022

Publisher's Note: MDPI stays neutral with regard to jurisdictional claims in published maps and institutional affiliations.

Copyright: (C) 2022 by the authors. Licensee MDPI, Basel, Switzerland. This article is an open access article distributed under the terms and conditions of the Creative Commons Attribution (CC BY) license (https:/ / creativecommons.org/licenses/by/ $4.0 /)$.
1 Department of Biotechnological and Applied Clinical Sciences, University of L'Aquila, 67100 L'Aquila, Italy; sabrina.cherubini@graduate.univaq.it (S.C.); mariagrazia.perilli@univaq.it (M.P.); gianfranco.amicosante@univaq.it (G.A.)

2 Department of Diagnostic and Public Health, Infectious Disease Section, University of Verona, 37134 Verona, Italy; annamaria.azzini@univr.it (A.M.A.); evelina.tacconelli@univr.it (E.T.)

3 Microbiology and Virology Unit, Department of Pathology and Diagnostic, Azienda Ospedaliera Universitaria Integrata di Verona, 37134 Verona, Italy; laura.maccacaro@aovr.veneto.it (L.M.); alda.bazaj@univr.it (A.B.); laura.naso@hotmail.it (L.N.); g.locascio@ausl.pc.it (G.L.C.)

4 Microbiology and Virology Unit, AUSL Piacenza, 29121 Piacenza, Italy

* Correspondence: alessandra.piccirilli@univaq.it

† Senior authors: M. Perilli and G. Lo Cascio.

$\ddagger \quad$ Members of LTCF-Veneto Working Group are listed in Acknowledgments.

\begin{abstract}
Long-term care facilities (LTCFs) are important reservoirs of antimicrobial-resistant (AMR) bacteria which colonize patients transferred from the hospital, or they may emerge in the facility as a result of mutation or gene transfer. In the present study, we characterized, from a molecular point of view, 43 E. coli strains collected from residents of LTCFs in Northern Italy. The most common lineage found was ST131, followed by sporadic presence of ST12, ST69, ST48, ST95, ST410 and ST1193. All strains were incubators of several virulence factors, with iss, sat, iha and senB being found in $84 \%, 72 \%$, $63 \%$ and $51 \%$ of $E$. coli, respectively. Thirty of the ST131 analyzed were of the O25b:H4 serotype and H30 subclone. The ST131 isolates were found to be mainly associated with IncF plasmids, CTX-M-1, CTX-M-3, CTX-M-15, CTX-M-27 and gyrA/parC/parE mutations. Metallo- $\beta$-lactamases were not found in ST131, whereas KPC-3 carbapenemase was found only in two ST131 and one ST1193. In conclusion, we confirmed the spread of extended-spectrum $\beta$-lactamase genes in E. coli ST131 isolated from colonized residents living inside LTCFs. The ST131 represents an incubator of fluoroquinolones, aminoglycosides and other antibiotic resistance genes in addition to different virulence factors.
\end{abstract}

Keywords: E. coli; WGS; antibiotic resistance genes; $\beta$-lactamases; virulome

\section{Introduction}

In the European Union/European Economic Area (EU/EEA) prior to December 2019, there were an estimated 2.9 million residents in 43,000 LTCFs, representing approximately $0.7 \%$ of the total population [1]. Reports from 2019 estimate that around $5.6 \%$ of the Italian population was potentially dependent, and $28.7 \%$ of them aged more than 65 in need of long-term care facilities (LTCFs) [1]. LTCFs are considered important reservoirs of antimicrobial-resistant (AMR) bacteria because of their colonization of residents discharged from the hospital, or they become colonized inside the facility as a result of antibiotic selective pressure [2-4]. The EU Centre for Disease Prevention and Control (ECDC) supports point prevalence surveys of healthcare-associated infections (HAIs) and antimicrobial use in European LTCFs [5]. This program monitors the burden of HAIs and antimicrobial 
resistance inside LTCFs, comparing data from different countries and aiding in the implementation of multimodal interventions to reduce them. Infections of the urinary tract, lower respiratory tract and gastroenteric tract caused by Enterobacterales, in particular Klebsiella pneumoniae and Escherichia coli, are the most frequent [6,7]; in particular, extra-intestinal pathogenic E. coli, resistant to numerous classes of antibiotics, is the leading cause of serious infections among LTCF residents, and the recent emergence and spread of extendedspectrum $\beta$-lactamases (ESBLs) and/or carbapenemase-producing Enterobacterales firmly reduced the therapeutic options. The aim of the present study was to characterize the resistome and virulome of E. coli strains isolated from residents of LTCFs in Veneto, a Northern Italian region. The study was assessed using a next-generation sequencing platform.

\section{Materials and Methods}

\subsection{Setting}

Between July 2018 and June 2019 we conducted a point prevalence survey to assess both the frequency of healthcare-related infections and the enteric colonization status by multi-antibiotic-resistant (MDR) Gram negative bacteria in the population of elderly residents of 27 long-term care facilities (LTCF) in the Veneto Region. Joining the survey was voluntary, but at least one facility for each province of the region was enrolled. The 27 facilities were not involved simultaneously but at different times based on the local Ethic Committee's approval, the willingness of local personnel to collaborate with researchers in the collection of the study-specific biological samples and the possibility of the reference microbiology laboratory to accept and process them. The survey was proposed to all patients who were hospitalized for at least $48 \mathrm{~h}$ and physically present in the facility at 8:00 a.m. of the survey day. Only patients who were able to provide written informed consent, or whose legal guardian consented, were enrolled. The survey was carried out in a single day for each facility. In the case of facilities with a high number of residents, the survey lasted several consecutive days, with the obligation to complete the evaluation of all subjects in each ward in a single day. For each enrolled patient, 118 variables were collected, including the type and etiology of concurrent infections and respective antibiotic therapy, any antibiotic treatments in the previous 3 months, hospital admissions and surgery in the previous 12 months and invasive medical devices in situ. For each enrolled host, a rectal swab was performed to assess the status of colonization by Gram negative MDR (Enterobacterales and non-fermenter Gram negatives producing ESBL and/or resistant to carbapenems).

\subsection{Strains Selection and Antimicrobial Susceptibility}

The strain selection was performed by inoculating the rectal swabs onto ChromID ESBL agar (bioMérieux, Marcy l'Etoile, France) and on Mac Conkey agar. Identification of the isolates was carried out in an automated Vitek2 System (bioMérieux, Marcy l'Etoile, France). Antimicrobial susceptibilities were performed on a Vitek2 system (version 9.02, bioMérieux, Marcy l'Etoile, France). The strains that showed resistance to carbapenems were also analyzed with an immunochromatographic lateral flow assay Carba5 (NG Biotech, Guipry, France). Resistance to antibiotics was interpreted according to the EUCAST criteria. The E. coli that showed susceptibility to third-generation cephalosporins and carbapenems were excluded from the study.

\subsection{DNA Extraction and Whole Genome Sequencing (WGS)}

Total genomic DNA was extracted from $1 \mathrm{~mL}$ of an overnight bacterial culture using a MagMAX Microbiome Ultra Nucleic Acid Isolation kit (Applied Biosystems and ThermoFisher Scientific, Monza, Italy) following the manufacturer's instructions. Whole genome sequencing (WGS) was performed using the Illumina Miseq platform with a $2 \times 300$ paired-end run. 


\subsection{Bioinformatics Analysis}

Quality control and sequences filtering were checked using DRAGEN FastQC + MultiQC v3.6.3 (https: / / basespace.illumina.com/apps/10562553/DRAGEN-FastQC-MultiQC, access date: 9 September 2021) and assembled with Velvet v1.2.10 (https://basespace. illumina.com/apps / 8556549/Velvet-de-novo-Assembly, access date: 9 September 2021) [8]. Acquired antimicrobial resistance genes and chromosomal point mutations ( $g y r A$, parC and parE genes) were identified using ResFinder 4.1 (https:/ / cge.cbs.dtu.dk/services/ ResFinder/, access date: 13 September 2021) [9] and virulence genes using VirulenceFinder 2.0 (https:/ / cge.cbs.dtu.dk/services/VirulenceFinder/, access date: 13 September 2021). Serotypes and fimH types were determined using SerotypeFinder 2.0 and FimTyper 1.0 (http:/ / genomicepidemiology.org/services/, access date: 14 September 2021) tools, respectively. Multilocus sequence typing (MLST) was performed using a BLAST-based approach [10]. The allele number of the seven housekeeping genes, adk, fumC, gyrB, icd, mdh, $\operatorname{pur} A$ and $\operatorname{rec} A$, and the Sequence Type (ST) of each isolate was determined by combining seven allelic profiles in MLST E. coli Atchman database (https://pubmlst.org/bigsdb?db= pubmlst_mlst_sqdef\&page=schemeInfo\&scheme_id=4, access date: 20 September 2021). PlasmidFinder 2.1 was used to detect the incompatibility groups of plasmids. Identified plasmids of the IncF, IncH1, IncH2, IncI1, IncN or IncA/C type were subtyped by pMLST 2.0 (https:/ / cge.cbs.dtu.dk/services/pMLST/, access date: 21 September 2021) [11].

\section{Results}

\subsection{Strains Selection and Antimicrobial Susceptibility}

Using a selective medium, 831 E. coli strains were isolated from 1981 residents of 27 LTCFs, and 43 strains collected from 13 LTCFs were randomly selected for WGS analysis. Overall, $100 \%$ of them were resistant to oxyimino-cephalosporins (cefotaxime and ceftazidime), $72 \%$ to ciprofloxacin and $21 \%$ to carbapenems (meropenem and/or imipenem), and $46 \%$ were also resistant to piperacillin-tazobactam association.

\subsection{WGS, MLST and Serotype}

E. coli strains were analyzed by whole-genome sequencing, which provides information about the sequence typing (MLST) of E. coli, serotypes, virulence genes, plasmid replicons, pMLST and antibiotic resistant genes (ARGs). The whole-genome size of the 43 E. coli ranged from 4.9 to $10 \mathrm{Mb}$. On the basis of the Achtman scheme, which considers $a d k$, fum $C, g y r B, i c d, m d h, p u r A$ and $\operatorname{rec} A$ housekeeping genes, the predominant ST found was ST131 (74\% of isolates) followed by ST12 (5\% isolates), ST69 (7\% of isolates), ST48 (2\% isolates), ST95 (2\% isolates), ST410 (2\% isolates) and ST1193 (7\% isolates) (Table 1). The serotype of each E. coli was also determined. Thirty ST131 isolates showed a O25b:H4 serotype with the fimbria variant fimH30 and two O61:H4 serotypes with fimH94. The ST12 belonged to the O4:H5 serotype with fimH5 and fimH240, the ST48 belonged to the O137:H4 with fimH54, the ST69 belonged to O15:H18 with fimH27 and O44-O77:H18 with fimH27, the ST95 belonged to O18:H7 with fimH15, the ST410 belonged to H21 with fimH24 and the ST1193 belonged to O75:H5 with fimH64. 
Table 1. Genome analysis of E. coli isolated from residents of 13 LTCFs (Northern Italian Region).

\begin{tabular}{|c|c|c|c|c|c|c|}
\hline $\begin{array}{l}\text { LTCFs } \\
\text { Code }\end{array}$ & $\begin{array}{l}\text { No. } \\
\text { Isolates }\end{array}$ & $\begin{array}{l}\text { Genome } \\
\text { Size (bp) }\end{array}$ & MLST * & Serotype & fimH & Virluence Genes \\
\hline \multirow[t]{9}{*}{ SSL_BL } & 2 & 5.360 .264 & ST69 & O15:H18 & 27 & $\begin{array}{l}\text { gad, air, lpf } A, \text { eilA, iss, } \\
\text { iha, sat, sen } B\end{array}$ \\
\hline & 1 & 5.064 .257 & ST131 & $\mathrm{O} 25 \mathrm{~b}: \mathrm{H} 4$ & 30 & sat, iss, iha, gad \\
\hline & 3 & 5.318 .811 & ST131 & O25b:H4 & 30 & sat, iss, cnf1, sen $B$ \\
\hline & 1 & 5.428 .542 & ST69 & $\begin{array}{l}\text { O17/O44- } \\
\text { O77:H18 }\end{array}$ & 27 & $\begin{array}{l}\text { eilA, gad, lpfA, sat, air, } \\
\operatorname{sen} B, \text { iss, iha }\end{array}$ \\
\hline & 1 & 5.506 .897 & ST131 & $\mathrm{O} 25 \mathrm{~b}: \mathrm{H} 4$ & 30 & $\operatorname{sen} B$, iss, sat, iha, cnf1 \\
\hline & 1 & 5.102 .820 & ST131 & $\mathrm{O} 25 \mathrm{~b}: \mathrm{H} 4$ & 30 & sat, iss, iha, gad \\
\hline & 1 & 5.257 .199 & ST95 & O18:H7 & 15 & $\begin{array}{l}\text { mchC, sen B, vat, ire } A \\
\text { iroN, iss, sfaS, gad, mchF }\end{array}$ \\
\hline & 1 & 5.278 .074 & ST12 & O4:H5 & 204 & $\begin{array}{l}\text { cnf1, iroN, mcmA, mchF, } \\
m c h C, \text { mchB, vat, iss, gad }\end{array}$ \\
\hline & 1 & 5.110 .437 & ST12 & O4:H5 & 5 & $\begin{array}{l}\text { cnf1, iroN, mcmA, mchF, } \\
m c h C, \text { mchB, vat, ireA }\end{array}$ \\
\hline ISRAA_TV & 1 & 5.117 .808 & ST131 & $\mathrm{O} 25 \mathrm{~b}: \mathrm{H} 4$ & 30 & $\operatorname{sen} B$, iss, sat, iha \\
\hline SAF_VE & 1 & 5.003 .689 & ST48 & O137:H4 & 54 & $m c h F$, gad, cma, iss, iroN \\
\hline \multirow[t]{2}{*}{ CRMC_VE } & 1 & 5.143 .042 & ST131 & $\mathrm{O} 25 \mathrm{~b}: \mathrm{H} 4$ & 30 & iha, $\operatorname{sen} B$, sat, iss \\
\hline & 1 & 5.126 .057 & ST131 & $\mathrm{O} 25 \mathrm{~b}: \mathrm{H} 4$ & 30 & sat, gad, iha, sen $B$, iss \\
\hline \multirow[t]{3}{*}{ CDS_RO } & 2 & 10.087.318 & ST131 & O61:H4 & 94 & ast $A$, iss, iha, sat \\
\hline & 1 & 5.158 .809 & ST1193 & O75:H5 & 64 & sat, iha \\
\hline & 1 & 5.151 .322 & ST131 & $\mathrm{O} 25 \mathrm{~b}: \mathrm{H} 4$ & 30 & iha, sat, iss \\
\hline \multirow[t]{3}{*}{ IPABMC_VI } & 1 & 5.362 .937 & ST131 & $\mathrm{O} 25 \mathrm{~b}: \mathrm{H} 4$ & 30 & sat, $\operatorname{sen} B$, ast $A$, iha, iss \\
\hline & 2 & 5.045 .886 & ST131 & $\mathrm{O} 25 \mathrm{~b}: \mathrm{H} 4$ & 30 & ast $A$, gad, iha, sen $B$, iss \\
\hline & 1 & 5.277 .401 & ST131 & $\mathrm{O} 25 \mathrm{~b}: \mathrm{H} 4$ & 30 & $\operatorname{sen} B$, iha, sat, ast $A$, iss \\
\hline \multirow[t]{2}{*}{ IPABRS_VI } & 2 & 5.238 .706 & ST131 & $\mathrm{O} 25 \mathrm{~b}: \mathrm{H} 4$ & 30 & gad, iha, sat, ast $A$, iss \\
\hline & 2 & 5.131 .308 & ST1193 & O75:H5 & 64 & vat \\
\hline \multirow[t]{4}{*}{ IPABRT_VI } & 2 & 4.999 .937 & ST131 & $\mathrm{O} 25 \mathrm{~b}: \mathrm{H} 4$ & 30 & sat, iss, senB \\
\hline & 2 & 5.120 .568 & ST131 & $\mathrm{O} 25 \mathrm{~b}: \mathrm{H} 4$ & 30 & sat, iss, $\operatorname{sen} B$, iha, gad \\
\hline & 1 & 4.865 .293 & ST131 & O25b:H4 & 30 & iss, sen $B$, iha \\
\hline & 1 & 5.080 .670 & ST131 & $\mathrm{O} 25 \mathrm{~b}: \mathrm{H} 4$ & 30 & iss, cnf1 \\
\hline \multirow[t]{2}{*}{ IPABSC_VI } & 2 & 5.165 .353 & ST131 & O25b:H4 & 30 & sat, iss, sen $B$, iha \\
\hline & 1 & 5.052 .997 & ST131 & $\mathrm{O} 25 \mathrm{~b}: \mathrm{H} 4$ & 30 & sat, iss, sen $B$, iha \\
\hline POBB_VR & 1 & 5.254 .036 & ST131 & $\mathrm{O} 25 \mathrm{~b}: \mathrm{H} 4$ & 30 & sat, iss, $\operatorname{sen} B$, iha, nfaE \\
\hline POVI_VR & 1 & 5.353 .306 & ST131 & $\mathrm{O} 25 \mathrm{~b}: \mathrm{H} 4$ & 30 & iss, iha, sat, cnf1 \\
\hline POSC_VR & 2 & 3.813 .125 & ST131 & $\mathrm{O} 25 \mathrm{~b}: \mathrm{H} 4$ & 30 & gad, iss, cnf1, iha \\
\hline \multirow[t]{2}{*}{ POCS_VR } & 1 & 4.907 .147 & ST410 & $\mathrm{H} 21$ & 24 & $g a d, \operatorname{lpf} A$ \\
\hline & 1 & 5.230 .571 & ST131 & $\mathrm{O} 25 \mathrm{~b}: \mathrm{H} 4$ & 30 & sat, iha, iss \\
\hline
\end{tabular}

For legal reasons, we used only the acronym of the 12 LTCFs. ${ }^{*}$ MLST: Multilocus sequence typing.

\subsection{Virulence Genes}

The virulence genes found in the E. coli isolates analyzed in this study were the following: sat (secreted autotransporter toxin), iha (adhesion-siderophore receptor), iss (increased serum survival), sen $B$ (plasmid encoded enterotoxin), gad (glutamate decarboxylase), ast $A$ (east-1 heat-stable toxin), lpfA (long polar fimbriae), air (enteroaggregative immunoglobulin repeat protein), eilA (Salmonella HilA homolog), cnf1 (cytotoxic necrotizing factor), vat (vacuolating autotransporter toxin), ireA (iron-regulated outer membrane virulence protein), iroN (salmchelin siderophore receptor), sfaS (S fimbriae), $n f a E$ (non fimbrial adhesion) and $m c m A, m c h B, m c h C$ and $m c h F$, which are the microcin $\mathrm{H} 47$ system virulence genes (Table 2). The increased serum survival (iss), secreted autotransporter toxin (sat), adhesion-siderophore receptor (iha) and plasmid encoded enterotoxin (senB) were found in $84 \%, 72 \%, 63 \%$ and $51 \%$ of E. coli, respectively. As shown in Table 1, in the E. coli ST12, ST69 and ST95, different virulence genes were found; on the contrary, only two virulence genes were identified in ST410 and ST1193. 
Table 2. Distribution of virulence genes in different $E$. coli.

\begin{tabular}{|c|c|c|}
\hline Virulence Gene & Target Class & Total No. $43 /(\%)$ \\
\hline air & Enteroaggregative immunoglobulin repeat protein & $2(5 \%)$ \\
\hline ast $A$ & East-1 heat stable toxin & $7(16 \%)$ \\
\hline cma & Colicin M & $2(5 \%)$ \\
\hline cnf1 & Cytotoxic necrotizing factor & $9(21 \%)$ \\
\hline eilA & Salmonella HilA homolog & $3(7 \%)$ \\
\hline gad & Glutamate decarboxylase & $10(23 \%)$ \\
\hline iha & Adhesion-siderophore receptor & $27(63 \%)$ \\
\hline ireA & Iron-regulated outer membrane virulence protein & $3(7 \%)$ \\
\hline iroN & Salmochelin siderophore receptor & $7(16 \%)$ \\
\hline iss & Increased serum survival & $36(84 \%)$ \\
\hline $\operatorname{lpf} A$ & Long polar fimbriae & $5(12 \%)$ \\
\hline$m c h B$ & Microcin H47 system & $3(7 \%)$ \\
\hline$m c h C$ & Microcin H47 system & $5(12 \%)$ \\
\hline$m c h F$ & Microcin H47 system & $7(16 \%)$ \\
\hline$m c m A$ & Microcin H47 system & $3(7 \%)$ \\
\hline$n f a E$ & non-fimbrial adhesion & $2(5 \%)$ \\
\hline sat & Secreted autotransporter toxin & $31(72 \%)$ \\
\hline $\operatorname{sen} B$ & Plasmid encoded enterotoxin & $22(51 \%)$ \\
\hline sfaS & S fimbriae & $2(5 \%)$ \\
\hline vat & Vacuolating autotransporter toxin & $7(16 \%)$ \\
\hline
\end{tabular}

\subsection{Plasmid Replicons and pMLST}

The different incompatibility plasmids IncI1, IncFII(29), IncFII(pRSB107), IncFIB, IncFIA, IncN, IncF, IncB/O/K/Z, IncQ1, IncX1, IncX3, IncX4, Col(MG828), Col156, Col8282 and ColRNAI were detected in all E. coli analyzed (Table 3). Each E. coli isolate harbored more than one type of plasmid. Overall, IncFII was the predominant plasmid, found in $84 \%$ of E. coli, followed by Col (Col(MG828), Col156, Col8282) in 76\%, IncFIA in 64\%, IncFIB in $60 \%$, ColRNAI in $40 \%$, IncN in $28 \%$, IncI 1 in $24 \%$, IncX (IncX4, IncX3 and IncX1) in $24 \%$ and $\mathrm{IncB} / \mathrm{O} / \mathrm{K} / \mathrm{Z}$ in $12 \%$ of $E$. coli. The pMLST showed that IncF was represented by several lineages such as fii_2, fii_24, fii_29, fia_1, fib_1, fib_10, F1:A2:B20, F1:A20:B31, F29:B10, F31:A20:B1, F1:A2, F4:A19, F2:A1, F1:A1:B10 and F46:A6:B47, whereas IncN plasmid was represented by the ST7 lineage. In detail, the pMLST of ST131 isolates revealed the presence of F1:A2:B20 (ten isolates), F1:A20:B31 (three isolates), F29:B10 (one isolate), F31:A20:B1 (one isolate), F1:A2 (one isolate), F2:A1 (three isolates) and F1:A1:B10 (two isolates) (Table 3).

Table 3. Resistome pattern of E. coli isolates from LTCFs in Northern Italian region.

\begin{tabular}{|c|c|c|c|c|c|}
\hline LTCFs Code & $\begin{array}{c}\text { No. } \\
\text { Isolates }\end{array}$ & Plasmid Replicons/pMLST & $\begin{array}{c}\text { B-Lactam } \\
\text { Resistant Genes }\end{array}$ & $\begin{array}{l}\text { Other Antibiotics } \\
\text { Resistant Genes }\end{array}$ & $\begin{array}{l}\text { Chromosomal } \\
\text { Point Mutation }\end{array}$ \\
\hline \multirow[t]{3}{*}{ SSL_BL } & 2 & $\begin{array}{l}\text { IncX4, IncI1, IncFII(29), IncN, } \\
\text { IncFIB(AP001918), Col156 } \\
\text { /IncF: fii_29, fib_10 } \\
\text { IncN: ST7 }\end{array}$ & $b^{b l a} a_{V I M-1}$ & $\begin{array}{l}\text { aac } A 4, \text { aad } A 1, \\
\operatorname{mph}(A), \text { sul1, } \\
d f r A 14, \text { qnrS1, } \\
\text { aac }\left(6^{\prime}\right) \text { Ib-cr, catB2 }\end{array}$ & $\begin{array}{l}\text { gyrA D87N } \\
\text { parC S80I }\end{array}$ \\
\hline & 1 & $\begin{array}{l}\text { Col(MG828),Col156, } \\
\text { IncFII(pRSB107), IncFIA, IncN } \\
\text { /IncF: F1:A2:B20 }\end{array}$ & $b l a_{C T X-M-3}$ & aadA5, sul1, dfrA17 & $\begin{array}{l}\text { gyrA S83L/D87N } \\
\text { parC S80I/E84V } \\
\text { parE I529L }\end{array}$ \\
\hline & 3 & $\begin{array}{l}\text { IncFII, Col156, IncFIB(AP001918), } \\
\text { IncFIA } \\
\text { /F1:A20:B31 }\end{array}$ & $\begin{array}{l}\text { bla }_{C T X-M-15} \\
\text { bla }_{\text {OXA-1 }}\end{array}$ & $\begin{array}{l}\text { aac }\left(6^{\prime}\right) I b-c r, \text { aad } A 5, \\
\text { aac }(3)-I I a, \operatorname{mph}(A), \\
\text { sul1, } \operatorname{dfr} A 17, \operatorname{tet}(A), \\
\text { catB4 }\end{array}$ & $\begin{array}{l}\text { gyrA S83L/D87N } \\
\text { parC S80I/E84V } \\
\text { parE I529L }\end{array}$ \\
\hline
\end{tabular}


Table 3. Cont.

\begin{tabular}{|c|c|c|c|c|c|}
\hline LTCFs Code & $\begin{array}{l}\text { No. } \\
\text { Isolates }\end{array}$ & Plasmid Replicons/pMLST & $\begin{array}{c}\text { B-Lactam } \\
\text { Resistant Genes }\end{array}$ & $\begin{array}{l}\text { Other Antibiotics } \\
\text { Resistant Genes }\end{array}$ & $\begin{array}{l}\text { Chromosomal } \\
\text { Point Mutation }\end{array}$ \\
\hline & 1 & $\begin{array}{l}\text { Col156, Col8282, IncI1, ColRNAI, } \\
\text { IncB/O/K/Z, IncFIB(AP001918), } \\
\text { IncFII(29), IncN } \\
\text { /IncF: F29:B10 } \\
\text { IncI1: arda_4, pill_6, repi1_1, } \\
\text { sogs_3, trba_6 } \\
\text { IncN: ST7 }\end{array}$ & $b l a_{V I M-1}, b l a_{T E M-1 B}$ & $\begin{array}{l}\operatorname{aph}\left(3^{\prime}\right)-X V \\
\operatorname{aac}\left(6^{\prime}\right) I b-c r, \operatorname{str} A, \\
\operatorname{str} B, \operatorname{aac} A 4, \text { aad } A 5, \\
\text { aadA1, } \operatorname{mph}(A), \\
\text { sul1, sul2, dfrA17, } \\
\text { qnrS1, catB2 }\end{array}$ & gyrA S83L \\
\hline & 1 & $\begin{array}{l}\text { IncFII, Col156, IncB/O/K/Z, } \\
\text { IncFIB(AP001918), IncFIA } \\
\text { /F31:A20:B1 }\end{array}$ & $\begin{array}{l}\text { bla }_{C T X-M-15} \\
\text { bla }_{\text {OXA-1 }}\end{array}$ & $\begin{array}{l}\operatorname{aac}\left(6^{\prime}\right) I b-c r \\
\operatorname{aac}(3)-I I a, c a t B 4\end{array}$ & $\begin{array}{l}\text { gyrA S83L/D87N } \\
\text { parC S80I/E84V } \\
\text { parE I529L }\end{array}$ \\
\hline & 1 & $\begin{array}{l}\text { IncFII(pRSB107), Col(MG828), } \\
\text { ColRNAI, IncFIA } \\
\text { /F1:A2 }\end{array}$ & $b l a_{C T X-M-27}$ & $\begin{array}{l}\operatorname{str} A, \text { strB, sul2, } \\
\operatorname{tet}(A), \text { aad } 55 \\
\operatorname{mph}(A), \text { sul1, } \\
d \operatorname{dr} A 17\end{array}$ & $\begin{array}{l}\text { gyrA S83L/D87N } \\
\text { parC S80I/E84V } \\
\text { parE I529L }\end{array}$ \\
\hline & 1 & $\begin{array}{l}\text { ColRNAI, IncFII(pCoo), IncN, } \\
\text { Col156 } \\
\text { /IncF: F29:B10 } \\
\text { IncN: ST7 }\end{array}$ & $b l a_{V I M-1}$ & $\begin{array}{l}\text { aac }\left(6^{\prime}\right) \operatorname{Ib-cr,aacA4}, \\
\operatorname{aacA1}, \operatorname{mph}(A), \text { sul1, } \\
\text { dfrA14, qnrS1, } \\
\text { catB2 }\end{array}$ & None \\
\hline & 1 & $\begin{array}{l}\text { IncFIB(AP001918), IncI1, IncQ1, } \\
\text { InFII }\end{array}$ & $\begin{array}{l}\text { bla }_{T E M-1 B} \\
\text { bla }_{C T X-M-1}\end{array}$ & $\begin{array}{l}\operatorname{aad} A 1, \operatorname{str} A, \operatorname{str} B \\
\operatorname{sul1}, \operatorname{sul} 2, d f r A 1 \\
\text { qnrS1, tet }(A)\end{array}$ & None \\
\hline & 1 & $\begin{array}{l}\text { IncFIA(HI1), IncFII, IncN } \\
\text { /IncF: F4:A19 } \\
\text { IncN: ST7 }\end{array}$ & $b l a_{V I M-1}$ & $\begin{array}{l}\operatorname{aac}\left(6^{\prime}\right) \operatorname{Ib}-c r, \text { aad } A 1, \\
\operatorname{aph}\left(3^{\prime}\right)-X V, \text { aacA4, } \\
m p h(A), \text { sul1, } \\
\text { dfrA14, qnrS1, } \\
\text { catB2 }\end{array}$ & None \\
\hline ISRAA_TV & 1 & $\begin{array}{l}\text { IncFII (pRSB107), IncB/O/K/Z, } \\
\text { IncFIA, IncFIB (AP001918), } \\
\text { Col(MG828), Col156 } \\
\text { /F1:A2:B20 }\end{array}$ & $b l a_{C T X-M-27}$ & $\begin{array}{l}\operatorname{str} A, \operatorname{str} B, \operatorname{sul} 2 \\
\operatorname{tet}(A)\end{array}$ & $\begin{array}{l}\text { gyrA S83L/D87N } \\
\text { parC S80I/E84V } \\
\text { parE I529L }\end{array}$ \\
\hline SAF_VE & 1 & $\begin{array}{l}\text { IncFII, IncI1, IncX1, } \\
\text { IncFIB(AP001918) } \\
\text { /IncF: fib_1, fii_24 }\end{array}$ & $b l a_{S H V-12}, b l a_{T E M-1 B}$ & $\begin{array}{l}\operatorname{str} A, \operatorname{str} B, \operatorname{aad} A 2, \\
\operatorname{aph}\left(3^{\prime}\right)-\operatorname{Ia}, \operatorname{lnu}(F), \\
\operatorname{qn} B 19, \operatorname{qnr} S 1 \\
\operatorname{tet}(B)\end{array}$ & None \\
\hline CRMC_VE & 1 & $\begin{array}{l}\text { IncFII(pRSB107), Col156, IncX4, } \\
\text { IncFIA, IncFIB (AP001918), } \\
\text { Col(MG828) } \\
\text { /F1:A2:B20 }\end{array}$ & $b l a_{C T X-M-27}$ & & $\begin{array}{l}\text { gyrA S83L/D87N } \\
\text { parC S80I/E84V } \\
\text { parE I529L }\end{array}$ \\
\hline & 1 & $\begin{array}{l}\text { IncFII(pRSB107),Col156, IncX4, } \\
\text { IncFIA, IncFIB (AP001918) } \\
\text { /F1:A2:B20 }\end{array}$ & $b l a_{C T X-M-27}$ & & $\begin{array}{l}\text { gyrA S83L/D87N } \\
\text { parC S80I/E84V } \\
\text { parE I529L }\end{array}$ \\
\hline \multirow[t]{2}{*}{ CDS_RO } & 2 & $\begin{array}{l}\text { Col(MG828), IncX4, IncI1, } \\
\text { Col8282,IncFIA,IncFII(pRSB107), } \\
\text { IncN, ColRNAI, Col(KPHS6), } \\
\text { p0111, Col156 /IncF: F2:A1 } \\
\text { IncI1: ST57 }\end{array}$ & $\begin{array}{l}\text { bla }_{T E M-1 B} \\
\text { bla }_{C T X-M-3} \\
\text { bla }_{O X A-1}\end{array}$ & $\begin{array}{l}\operatorname{aph}\left(3^{\prime}\right)-I I a, \\
\operatorname{aac}\left(6^{\prime}\right)-I b-c r, c a t B 4\end{array}$ & None \\
\hline & 1 & $\begin{array}{l}\text { Col156, Col(MG828), IncFIA, } \\
\text { IncFII } \\
\text { /F2:A1 }\end{array}$ & $\begin{array}{l}\text { bla }_{C T X-M-15} \\
\text { bla }_{O X A-1}\end{array}$ & $a a c\left(6^{\prime}\right)-I b-c r, c a t B 4$ & $\begin{array}{l}\text { gyrA S83L/D87N } \\
\operatorname{parC~S80I/E84V~} \\
\operatorname{parE~I529L~}\end{array}$ \\
\hline
\end{tabular}


Table 3. Cont.

\begin{tabular}{|c|c|c|c|c|c|}
\hline LTCFs Code & $\begin{array}{l}\text { No. } \\
\text { Isolates }\end{array}$ & Plasmid Replicons/pMLST & $\begin{array}{c}\text { B-Lactam } \\
\text { Resistant Genes }\end{array}$ & $\begin{array}{l}\text { Other Antibiotics } \\
\text { Resistant Genes }\end{array}$ & $\begin{array}{l}\text { Chromosomal } \\
\text { Point Mutation }\end{array}$ \\
\hline & 1 & $\begin{array}{l}\text { IncFIB(pQil),Col(BS512), IncFIA, } \\
\text { IncFIB(AP001918) } \\
\text { /IncF: A1:B10 }\end{array}$ & $\begin{array}{l}\text { bla }_{T E M-1 A}, b l a_{K P C-3} \\
\text { bla }_{O X A-9}\end{array}$ & aac(3)-IId & $\begin{array}{l}\text { gyrA S83L/D87N } \\
\text { parC S80I } \\
\text { parE L416F }\end{array}$ \\
\hline \multirow[t]{3}{*}{ IPABMC_VI } & 1 & $\begin{array}{l}\text { Col(MG828), IncX4, IncFII(29), } \\
\text { Col156, ColRNAI, } \\
\text { IncFIB(AP001918) /F29:B10 }\end{array}$ & $\begin{array}{l}\text { bla }_{\text {TEM-1B }} \\
\text { bla }_{C T X-M-15}\end{array}$ & $\begin{array}{l}\text { aac(3)-Id, str } A, \operatorname{str} B \\
\text { aad } A 5, \operatorname{mph}(A), \\
\text { sul1, sul2, dfrA17, } \\
\operatorname{tet}(A)\end{array}$ & $\begin{array}{l}\text { gyrA S83L/D87N } \\
\text { parC S80I/E84V } \\
\text { parE I529L }\end{array}$ \\
\hline & 2 & $\begin{array}{l}\text { IncFII(29), IncFIB(AP001918), } \\
\text { Col8282, ColRNAI, Col156 } \\
\text { /F29:B10 }\end{array}$ & $\begin{array}{l}\text { bla }_{C T X-M-15} \\
\text { bla }_{T E M-1 B}\end{array}$ & $\begin{array}{l}\operatorname{str} A, \operatorname{aac}(3)-I I d, \\
\operatorname{str} B, \operatorname{aad} A 5, \\
\operatorname{mph}(A), \operatorname{sul} 2, \text { sul1, } \\
\operatorname{dfr} A 17, \operatorname{tet}(A)\end{array}$ & $\begin{array}{l}\text { gyrA S83L/D87N } \\
\text { parC S80I/E84V } \\
\text { parE I529L }\end{array}$ \\
\hline & 1 & $\begin{array}{l}\text { IncFII(29), IncFIB(AP001918), } \\
\text { Col156, ColRNAI, Col8282 } \\
\text { /IncF: F29:B10 }\end{array}$ & $\begin{array}{l}\text { bla }_{T E M-1 B} \\
\text { bla }_{C T X-M-15} \\
\text { bla }_{V I M-1}\end{array}$ & $\begin{array}{l}\text { aph }\left(3^{\prime}\right)-X V, \text { aacA4, } \\
\text { aad } A 1, \operatorname{aac}(3)-I I d, \\
\operatorname{str} A, \operatorname{str} B, \operatorname{mph}(A), \\
\text { sul1, sul2, } \\
\text { aac }\left(6^{\prime}\right) I b-c r, d f r A 14, \\
\operatorname{dfr} A 17, \text { qnrS1, } \\
\operatorname{tet}(A), \operatorname{catB2}\end{array}$ & $\begin{array}{l}\text { gyrA S83L/D87N } \\
\text { parC S80I/E84V } \\
\text { parE I529L }\end{array}$ \\
\hline \multirow[t]{2}{*}{ IPABRS_VI } & 2 & $\begin{array}{l}\text { IncFII, IncFIA, Col(MG828) } \\
\text { /F2:A1 }\end{array}$ & $\begin{array}{l}\text { bla }_{C T X-M-15} \\
\text { bla }_{T E M-1 B}\end{array}$ & & $\begin{array}{l}\text { gyrA S83L/D87N } \\
\text { parC S80I/E84V } \\
\text { parE I529L }\end{array}$ \\
\hline & 2 & $\begin{array}{l}\text { IncFIA, Col (MG828), IncFIB } \\
\text { (AP001918), Col156 } \\
\text { /F1:A1:B10 }\end{array}$ & $\begin{array}{l}\text { bla }_{T E M-1 A} \\
\text { bla }_{S H V-12}\end{array}$ & $\begin{array}{l}\operatorname{mph}(A), \text { sul2, sul1, } \\
\operatorname{dfr} A 17, \text { qnrB19, } \\
\operatorname{tet}(A)\end{array}$ & $\begin{array}{l}\text { gyrA S83L/D87N } \\
\text { parC S80I } \\
\text { parE L416F }\end{array}$ \\
\hline \multirow[t]{4}{*}{ IPABRT_VI } & 2 & $\begin{array}{l}\text { IncFII(AP001918), } \\
\text { IncFII(pRSB107), Col8282, IncFIA, } \\
\text { Col156, Col(MG828) } \\
\text { /F1:A2:B20 }\end{array}$ & $b l a_{C T X-M-27}$ & $\begin{array}{l}\operatorname{str} B, \text { aad } A 5, \operatorname{str} A, \\
\operatorname{sul} 1, \operatorname{sul} 2, d f r A 17 \\
\operatorname{tet}(A)\end{array}$ & $\begin{array}{l}\text { gyrA S83L/D87N } \\
\text { parC S80I/E84V } \\
\text { parE I529L }\end{array}$ \\
\hline & 2 & $\begin{array}{l}\text { IncFII(AP001918), } \\
\text { IncFII(pRSB107), Col8282, IncFIA, } \\
\text { Col156, Col(MG828) } \\
\text { /F1:A2:B20 }\end{array}$ & $b l a_{C T X-M-27}$ & $\begin{array}{l}\operatorname{str} A, \text { aad } A 5, \operatorname{str} B \\
\operatorname{mph}(A), \operatorname{sul} 1, \operatorname{sul} 2, \\
\operatorname{dfr} A 17, \operatorname{tet}(A)\end{array}$ & $\begin{array}{l}\text { gyrA S83L/D87N } \\
\text { parC S80I/E84V } \\
\text { parE I529L }\end{array}$ \\
\hline & 1 & $\begin{array}{l}\text { IncFII(pRSB107), Col156, } \\
\text { IncFIB(AP001918), IncFIB(pQil), } \\
\text { IncFIA } \\
\text { /IncF: F1:A2:B20 }\end{array}$ & $\begin{array}{l}\text { bla } \\
\text { bla }_{K P C-3}, \text { bla } \\
\text { bla }_{\text {CTX-M-27 }}\end{array}$ & $\begin{array}{l}\operatorname{aac}(3)-I I a \\
a a c\left(6^{\prime}\right) I b-c r, d f r A 14 \\
q n r B 66, c a t B 4\end{array}$ & $\begin{array}{l}\text { gyrA S83L/D87N } \\
\text { parC S80I/E84V } \\
\text { parE I529L }\end{array}$ \\
\hline & 1 & $\begin{array}{l}\text { IncFIA,IncFIB(AP001918), IncFII, } \\
\text { ColRNAI } \\
\text { /F31:A20:B1 }\end{array}$ & $b l a_{C T X-M-15}, b l a_{O X A-1}$ & catB4 & $\begin{array}{l}\text { gyrA S83L/D87N } \\
\text { parC S80I/E84V } \\
\text { parE I529L }\end{array}$ \\
\hline \multirow[t]{2}{*}{ IPABSC_VI } & 2 & $\begin{array}{l}\text { IncFIB(AP001918), } \\
\text { IncFII(pRSB107), Col8282, IncFIA, } \\
\text { ColRNAI, Col156, IncI1 } \\
\text { /F1:A2:B20 }\end{array}$ & $b l a_{C T X-M-27}$ & $\begin{array}{l}\operatorname{str} B, \operatorname{aad} A 5, \operatorname{str} A \\
\operatorname{mph}(A), \operatorname{sul} 1, \operatorname{sul} 2, \\
\operatorname{dfr} A 17, \operatorname{tet}(A)\end{array}$ & $\begin{array}{l}\text { gyrA S83L/D87N } \\
\text { parC S80I/E84V } \\
\text { parE I529L }\end{array}$ \\
\hline & 1 & $\begin{array}{l}\text { IncFIB(AP001918), } \\
\text { IncFII(pRSB107), IncFIA, Col156 } \\
\text { /F1:A2:B20 }\end{array}$ & $b l a_{C T X-M-27}$ & $\begin{array}{l}\operatorname{str} B, \operatorname{aad} A 5, \operatorname{str} A \\
\operatorname{mph}(A), \operatorname{sul} 1, \operatorname{sul} 2, \\
\operatorname{dfr} A 17, \operatorname{tet}(A)\end{array}$ & $\begin{array}{l}\text { gyrA S83L/D87N } \\
\text { parC S80I/E84V } \\
\text { parE I529L }\end{array}$ \\
\hline POBB_VR & 1 & $\begin{array}{l}\text { IncFIA, IncFIB(AP001918), } \\
\text { IncFII(pRSB107), Col8282, IncN, } \\
\text { Col156, ColRNAI } \\
\text { /F1:A2:B20 } \\
\text { IncN: ST7 }\end{array}$ & $\begin{array}{l}\text { bla }_{C T X-M-15} \\
\text { bla }_{T E M-1 B}\end{array}$ & 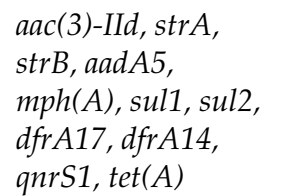 & $\begin{array}{l}\text { gyrA S83L/D87N } \\
\text { parC S80I/E84V } \\
\text { parE I529L }\end{array}$ \\
\hline
\end{tabular}


Table 3. Cont.

\begin{tabular}{|c|c|c|c|c|c|}
\hline LTCFs Code & $\begin{array}{c}\text { No. } \\
\text { Isolates }\end{array}$ & Plasmid Replicons/pMLST & $\begin{array}{c}\text { B-Lactam } \\
\text { Resistant Genes }\end{array}$ & $\begin{array}{l}\text { Other Antibiotics } \\
\text { Resistant Genes }\end{array}$ & $\begin{array}{l}\text { Chromosomal } \\
\text { Point Mutation }\end{array}$ \\
\hline POVI_VR & 1 & $\begin{array}{l}\text { IncFIA, IncFIB(AP001918), IncFII, } \\
\text { ColRNAI } \\
\text { /F31:A20:B1 }\end{array}$ & $b l a_{C T X-M-15}, b l a_{O X A-1}$ & $\begin{array}{l}\operatorname{aac}(3)-I I a, \\
a a c\left(6^{\prime}\right) I b-c r, \operatorname{tet}(A), \\
\text { catB4 }\end{array}$ & $\begin{array}{l}\text { gyrA S83L/D87N } \\
\text { parC S80I/E84V } \\
\text { parE I529L }\end{array}$ \\
\hline POSC_VR & 2 & $\begin{array}{l}\text { IncFIB(AP001918), ColRNAI, } \\
\text { IncI1, IncFII, IncFIA } \\
\text { /F36:A20:B1 }\end{array}$ & $b^{b l a_{C T X-M-15}, b l a_{O X A-1}}$ & $\begin{array}{l}\text { aac (3)-IIa, } \\
\operatorname{aac}\left(6^{\prime}\right) I b-c r, \operatorname{tet}(A), \\
\text { catB4 }\end{array}$ & $\begin{array}{l}\text { gyrA S83L/D87N } \\
\text { parC S80I/E84V } \\
\text { parE I529L }\end{array}$ \\
\hline \multirow[t]{2}{*}{ POCS_VR } & 1 & $\begin{array}{l}\text { IncX3, ColRNAI, IncFIB(pB171), } \\
\text { IncI1, IncP1, FIA (pBK30683), } \\
\text { IncFIC(FII) } \\
\text { /F46:A6:B47 }\end{array}$ & $b l a_{C M Y-42}, b l a_{N D M-4}$ & $\operatorname{tet}(B)$ & $\begin{array}{l}\text { gyrA S83L/D87N } \\
\text { parC S80I } \\
\text { parE S458A }\end{array}$ \\
\hline & 1 & $\begin{array}{l}\text { IncFII(29), IncFIB(AP001918), } \\
\text { ColRNAI, IncFIB(pQil) } \\
\text { /IncF: F29:B10 }\end{array}$ & $\begin{array}{l}\text { bla }_{C T X-M-15}, b l a_{O X A-9} \\
\text { bla }_{T E M-1 A}, b l a_{K P C-3} \\
\text { bla }_{O X A-1}\end{array}$ & $a a c\left(6^{\prime}\right)-I b-c r$ & $\begin{array}{l}\text { gyrA S83L/D87N } \\
\text { parC S80I/E84V } \\
\text { parE I529L }\end{array}$ \\
\hline
\end{tabular}

\subsection{Antibiotic Resistance Genes (ARGs)}

The aminoglycoside resistance genes aadA1, aadA2, aadA5, aacA1, aacA4, aac(3)-IId, $\operatorname{aph}\left(3^{\prime}\right)-X V$, aph $\left(3^{\prime}\right)-I a$ and $a p h\left(3^{\prime}\right)-I I a$ and the bi-functional gene $a a c\left(6^{\prime}\right) I b-c r$ were found in all E. coli analyzed (Table 3). Fluoroquinolone resistance was plasmid-mediated by the presence of $q n r$ elements. In particular, the $q n r S 1$ was identified in ten E. coli, whereas qnrB19 and qnrB66 were found in two and one isolates, respectively. In one isolate of E. coli, the simultaneous presence of $q n r S 1$ and $q n r B 19$ was identified. The most common mechanism of sulfamethoxazole/trimethoprim resistance was represented by the acquisition of dihydrofolate reductase $d f r A 1$ (1 isolate), $d f r A 14$ (8 isolates) and $d f r A 17$ (19 isolates). Other antibiotic resistance genes were the following: $m p h A$ (macrolide resistance) detected in 23 E. coli, sul1/sul2 (suphonamide resistance) detected in 26 isolates, catB2/catB4 (chloramphenicol resistance) detected in 17 isolates, $\operatorname{str} A / \operatorname{str} B$ (streptomycin resistance) detected in 17 isolates and tet $(A) / \operatorname{tet}(B)$ (tetracycline resistance) detected in 22 isolates. The lincosamide nucleotidyltransferase gene $(\ln u(F))$ was found in one ST48 isolate. All E. coli strains produced one or more $\beta$-lactamases belonging to molecular classes $A, B, C$ and $D$. Consistent with the results in this study, the ESBLs were the most widespread, and they included enzymes of the CTX-M-1 group (CTX-M-1, CTX-M-3 and CTX-M-15) in 21 isolates, CTX-M-27 (belonging to CTX-M-9 group) in 12 isolates and SHV-12 in 3 isolates (Table 4). The CTX-M enzymes were found only in ST131 strains. Overall, 37 out 43 E. coli (87\%) were positive to ESBLs. The VIM-1 enzyme was found in three ST69, one ST12 and one ST95. The E. coli ST410 produced the metallo- $\beta$-lactamase NDM-4 and CMY-42 class C enzyme. The KPC-3 carbapenemase was identified in three isolates, specifically two ST131 and one ST1193. The OXA-1 and OXA-9 were found in 12 and 3 isolates, respectively, and in association with other $\beta$-lactamase genes (Table 4 ).

\subsection{Chromosomal Fluoroquinolones Resistance}

Resistance to fluoroquinolones was also mediated by mutations in $g y r A$, parC and parE. As shown in Table 3, the S83L/D87N in $g y r A, S 80 \mathrm{I} / \mathrm{E} 84 \mathrm{~V}$ in parC and I529L in parE were the most common substitutions found in twenty-nine ST131. The S83L/D87N in gyrA, S80I in parC and L416F in parE mutations were found in three ST1193. The S83L/D87N in gyrA, S80I in parC and S458A in parE mutations were identified in one ST410. Two isolates of E. coli ST69 showed the S83L and D87N mutations in gyrA and S80I in parC. 
Table 4. Distribution of $\beta$-lactamase genes among E. coli strains.

\begin{tabular}{|c|c|c|c|c|c|c|c|}
\hline E. coli & ST12 & ST48 & ST69 & ST95 & ST131 & ST410 & ST1193 \\
\hline Total No. & 2 & 1 & 3 & 1 & 32 & 1 & 3 \\
\hline$b l a_{\mathrm{TEM}-1}$ & 1 & 1 & 1 & - & 9 & - & 3 \\
\hline$b l a_{\mathrm{CTX}-\mathrm{M}-1}$ & 1 & - & - & - & - & - & - \\
\hline$b l a_{\mathrm{CTX}-\mathrm{M}-3}$ & - & - & - & - & 3 & - & - \\
\hline bla $a_{\mathrm{CTX}-\mathrm{M}-15}$ & - & - & - & - & 17 & - & - \\
\hline$b l a_{\mathrm{CTX}-\mathrm{M}-27}$ & - & - & - & - & 12 & - & - \\
\hline$b l a_{\mathrm{SHV}-12}$ & - & 1 & - & - & - & - & 2 \\
\hline$b l a_{\mathrm{KPC}-3}$ & - & - & - & - & 2 & - & 1 \\
\hline$b l a_{\mathrm{NDM}-4}$ & - & - & - & - & - & 1 & - \\
\hline$b l a_{\mathrm{VIM}-1}$ & 1 & - & 3 & 1 & - & - & - \\
\hline$b l a_{\mathrm{CMY}-42}$ & - & - & - & - & - & 1 & - \\
\hline$b l a_{\mathrm{OXA}-1}$ & - & - & - & - & 13 & - & - \\
\hline$b l a_{\text {OXA-9 }}$ & - & - & - & - & 2 & - & 1 \\
\hline
\end{tabular}

\section{Discussion}

Herein, we used NGS-technology to investigate the resistome, virulome and genetic diversity of multi-drug resistant E. coli collected from 13 LTCFs in the Veneto region. The majority of E. coli analyzed belongs to the ST131 lineage. From a clinical point of view, ST131 is an extra-intestinal pathogenic bacterium which lives in the digestive tract, but it could also mediate its pathogenicity in the urinary tract and in the blood stream [12]. Almost all ST131 (30 out of 32) analyzed were of the O25b:H4 serotype and H30 subclone. The fimH allele, encoding for the virulence factor used by bacteria to attach the host tissue, has been used to phylogenetically classify the ST131 isolates in three clades (A, B, C), being also based on antibiotic resistance genes $[13,14]$. As reported by Pitout et al., ST131 clades $\mathrm{C} 1$ and $\mathrm{C} 2$ are fluoroquinolone resistant, and $\mathrm{C} 2$ has a strong association with CTX-M production [14]. Several studies have proven that the most prevalent subclonal lineage of E. coli ST131 is fimH30, which is also associated with a specific mutation in gyrA and parC, conferring chromosomal resistance to fluoroquinolones $[15,16]$. Some $E$. coli lineages were identified only in one LTCF; this is the case for ST12, ST69 and ST95, found only in SSL_BL, the ST48 in SAF_VE and ST410 in POCS_VR. The ST410 lineage seems to be phylogenetically older than ST131, but because of its virulence profile, ST131 is globally distributed in several environments over the world [17]. The feature of ST410 strains, in contrast to ST131, is the resistance to carbapenems [17]. In the present study, the ST410-H24 strain showed resistance to carbapenems, producing NDM-4 metallo- $\beta$-lactamase, and to fluoroquinolones via plasmid qnrS1 and to chromosomes by mutations in $g y r A$, parC and parE. The ST131 isolates were found to be associated with IncF plasmids, ESBLs (CTXM-1-group, CTX-M-27) and chromosomal resistance to fluoroquinolone (gyrA/parC/parE mutations) [18]. They also possessed $a a c\left(6^{\prime}\right)-I b-c r, c a t B 4$, OXA-1/OXA-9 and other ARGs which reduced susceptibility to aminoglycoside, chloramphenicol, oxacillin and other classes of antibiotics. In ST131, we found the predominance of CTX-M variants (94\% of isolates) followed by OXA-1/OXA-9 (47\% of isolates). As previously reported, CTX-M-15 and CTX-M-27 alleles are associated with the ST131 clade C2 and ST131 clade C1 [19-21]. The CTX-M-27 is predominantly associated with the F1:A2:B20 replicon, as reported in other ST131 strains isolated worldwide [22]. The CTX-M-27 belongs to subgroup CTX-M-9, and it showed two amino acid substitutions from CTX-M-9 (A231V and D240G) and one from CTX-M-14 (D240G). CTX-M-27 was found, not only in clinical strains, but also in bacteria isolated from food-producing animals, livestock and environment [23-25]. Metallo$\beta$-lactamases were not found in ST131, and this is in agreement with results reported in different countries [13]. Similarly, the KPC-3 carbapenemase was found only in three E. coli strains (ST131 and ST1193) collected in CDS_RO, IPABRT_VI and PCS_VR LTCFs. In a previous paper where we characterized the resistome of $K$. pneumoniae isolates, we found, in the same LTCFs, KPC-3 producing K. pneumoniae [26]. The E. coli and K. pneumoniae strains harbored the same IncFIB(pQil) plasmid. We speculate that, in the same LTCF, a transfer of 
the $b_{1} a_{\mathrm{KPC}-3}$ gene, presumably located in IncFIB(pQil) plasmid, may have occurred between E. coli and K. pneumoniae.

\section{Conclusions}

In this study, we confirmed the spread of extended-spectrum $\beta$-lactamases and carbapenemases in E. coli isolated from colonized residents living inside LTCFs. In particular, the CTX-M-enzymes belonging to sub-groups 1 and 9 represent the ESBLs mainly found in our strains. In the past decade, the CTX-M-enzymes have been increasingly detected in E. coli worldwide. A low percentage of carbapenemases, including KPC-, VIM- and NDM-variants, was identified in E. coli collected in the LTCFs included in this survey. The ST131 represents an incubator of fluoroquinolones, aminoglycosides and other antibiotic resistance genes and, in addition, different virulence factors. ST131 is composed by five clades (A, B, C0, C1 and C2), and clade C seems to be more fit than the others. The clade $\mathrm{C}$ showed resistance to fluoroquinolones and was able to acquire IncF plasmids, giving to $E$. coli a rapid and continual adaptation to different environments. Emerging evidence worldwide suggests that LTCFs are important reservoirs for ARGs' dissemination. The presence of antimicrobial-resistant pathogens in LTCFs has serious consequences not only for residents but also for the LTCF as an organization, both in terms of internal strategies to contain and possibly reduce these pathogens and the acceptance of colonized patients from the hospital.

Author Contributions: Conceptualization, A.P. and M.P.; methodology, A.M.A., A.P., S.C., L.M., A.B. and L.N.; investigation, A.P., S.C. and A.M.A.; data curation, M.P., G.L.C. and A.M.A.; writingoriginal draft preparation, M.P. and A.M.A.; writing-review and editing, M.P., A.P., G.L.C. and A.M.A.; visualization, E.T.; supervision, M.P., G.A. and E.T.; project administration, A.P., G.L.C. and A.M.A.; funding acquisition, A.M.A., G.A. and M.P. All authors have read and agreed to the published version of the manuscript.

Funding: This research was funded by Regione Veneto, DGR 1656; 17/10/2017 and University of L'Aquila internal funds (07_PROGETTO_RICERCA_ATENEO).

Institutional Review Board Statement: Not applicable.

Informed Consent Statement: Not applicable.

Data Availability Statement: Data sharing not applicable.

Acknowledgments: The authors thank Giuseppe Cornaglia for having strongly supported this study with his scientific enthusiasm. Thanks to the LTCF Veneto working group, composed of Giorgia Be, Nicola Salerno, Lorenza Lambertenghi and Ilaria Coledan (Infectious Disease Section, Department of Diagnostic and Public Health, University of Verona, Italy) for the collection of biological samples.

Conflicts of Interest: The authors declare no conflict of interest.

\section{References}

1. European Centre for Disease Prevention and Control. Surveillance of COVID-19 in Long-Term Care Facilities in the EU/EEA; ECDC: Stockholm, Sweden, 2021.

2. Moro, M.L.; Gagliotti, C. Antimicrobial resistance and stewardship in long-term care facilities. Future Microbiol. 2013, 8, 1011-1025. [CrossRef]

3. Strausbaugh, L.J.; Joseph, C.L. The burden of infection in long-term care. Infect Control. Hosp. Epidemiol. 2000, 21, 674-679. [CrossRef]

4. Giufrè, M.; Ricchizzi, E.; Accogli, M.; Barbanti, F.; Monaco, M.; Pimentel de Araujo, F.; Farina, C.; Fazii, P.; Mattei, R.; Sarti, M.; et al. Colonization by multidrug-resistant organisms in long-term care facilities in Italy: A point-prevalence study. Clin. Microbiol. Infect. 2017, 23, 961-967. [CrossRef]

5. European Centre for Disease Prevention and Control. Protocol for Validation of Point Prevalence Surveys of Healthcare-Associated Infections and Antimicrobial Use in European Long-Term Care Facilities-2016-2017 Version 1.1; ECDC: Stockholm, Sweden, 2016.

6. Rosello, A.; Hayward, A.C.; Hopkins, S.; Horner, C.; Ironmonger, D.; Hawkey, P.M.; Deeny, S.R. Impact of long-term care facility residence on the antibiotic resistance of urinary tract Escherichia coli and Klebsiella. J. Antimicrob. Chemother. 2017, 72, 1184-1192. [PubMed]

7. Falcone, M.; Paul, M.; Yahav, D.; Orlando, G.; Tiseo, G.; Prendki, V.; Güerri-Fernández, R.; Gavazzi, G.; Mutters, N.T.; Cookson, B.; et al. Antimicrobial consumption and impact of antimicrobial stewardship programmes in long-term care facilities. Clin. Microbiol. Infect. 2019, 25, 562-569. [CrossRef] 
8. Zerbino, D.R. Using the Velvet de novo assembler for short-read sequencing technologies. Curr. Protoc. Bioinform. 2010, 11, 11-15. [CrossRef]

9. Zankari, E.; Hasman, H.; Cosentino, S.; Vestergaard, M.; Rasmussen, S.; Lund, O.; Aarestrup, F.M.; Larsen, M.V. Identification of acquired antimicrobial resistance genes. J. Antimicrob. Chemother. 2012, 67, 2640-2644. [CrossRef] [PubMed]

10. Larsen, M.V.; Cosentino, S.; Rasmussen, S.; Friis, C.; Hasman, H.; Marvig, R.L.; Jelsbak, L.; Sicheritz-Pontén, T.; Ussery, D.W.; Aarestrup, F.M.; et al. Multilocus sequence typing of total-genome-sequenced bacteria. J. Clin. Microbiol. 2012, 50, $1355-1361$. [CrossRef] [PubMed]

11. Carattoli, A.; Zankari, E.; García-Fernández, A.; Voldby Larsen, M.; Lund, O.; Villa, L.; Møller Aarestrup, F.; Hasman, H. In silico detection and typing of plasmids using PlasmidFinder and plasmid multilocus sequence typing. Antimicrob. Agents Chemother. 2014, 58, 3895-3903. [CrossRef] [PubMed]

12. Manges, A.R.; Geum, H.M.; Guo, A.; Edens, T.J.; Fibke, C.D.; Pitout, J.D.D. Global Extraintestinal pathogenic Escherichia coli (ExPEC) lineages. Clin. Microbiol. Rev. 2019, 32, e00135-18. [CrossRef]

13. Nicolas-Chanoine, M.H.; Bertrand, X.; Madec, J.Y. Escherichia coli ST131, an intriguing clonal group. Clin. Microbiol. Rev. 2014, 27, 543-574. [CrossRef] [PubMed]

14. Pitout, J.D.D.; Finn, T.J. The evolutionary puzzle of Escherichia coli ST131. Infect. Genet. Evol. 2020, 81, 104265. [CrossRef]

15. Johnson, J.R.; Porter, S.B.; Zhanel, G.; Kuskowski, M.A.; Denamur, E. Virulence of Escherichia coli clinical isolates in a murine sepsis model in relation to sequence type ST131 status, fluoroquinolone resistance, and virulence genotype. Infect. Immun. 2012, 80, 1554-1562. [CrossRef]

16. Petty, N.K.; Ben Zakour, N.L.; Stanton-Cook, M.; Skippington, E.; Totsika, M.; Forde, B.M.; Phan, M.D.; Moriel, D.G.; Peters, K.M.; Davies, M. Global dissemination of a multidrug resistant Escherichia coli clone. Proc. Natl. Acad. Sci. USA 2014, 111, 5694-5699. [CrossRef]

17. Roer, L.; Overballe-Petersen, S.; Hansen, F.; Schønning, K.; Wang, M.; Røder, B.L.; Hansen, D.S.; Justesen, U.S.; Andersen, L.P.; Fulgsang-Damgaard, D.; et al. Escherichia coli sequence type 410 is causing new international high-risk clones. $m S p h e r e ~ 2018,3$, e00337-18. [CrossRef] [PubMed]

18. Matsumura, Y.; Yamamoto, M.; Nagao, M.; Ito, Y.; Takakura, S.; Ichiyama, S. Association of fluoroquinolone resistance, virulence genes, and IncF plasmids with extended-spectrum- $\beta$-lactamase-producing Escherichia coli sequence type 131 (ST131) and ST405 clonal groups. Antimicrob. Agents Chemother. 2013, 57, 4736-4742. [CrossRef]

19. Matsumura, Y.; Johnson, J.R.; Yamamoto, M.; Nagao, M.; Tanaka, M.; Takakura, S.; Ichiyama, S. CTX-M-27- and CTX-M-14producing, ciprofloxacin-resistant Escherichia coli of the H30 subclonal group within ST131 drive a Japanese regional ESBL epidemic. J. Antimicrob. Chemother. 2015, 70, 1639-1649. [CrossRef]

20. Matsumura, Y.; Pitout, J.D.D.; Gomi, R.; Matsuda, T.; Noguchi, T.; Yamamoto, M.; Peirano, G.; Devinney, R.; Bradford, P.A.; Motyl, M.R.; et al. Global Escherichia coli sequence type 131 clade with blaCTX-M-27 gene. Emerg. Infect. Dis. 2016, 22, 1900-1907. [CrossRef]

21. Seiffert, S.N.; Hilty, M.; Kronenberg, A.; Droz, S.; Perreten, V.; Endimiani, A. Extended-spectrum cephalosporin-resistant Escherichia coli in community, specialized outpatient clinic and hospital settings in Switzerland. J. Antimicrob. Chemother. 2013, 68, 2249-2254. [CrossRef] [PubMed]

22. Matsuo, N.; Nonogaki, R.; Hayashi, M.; Wachino, J.I.; Suzuki, M.; Arakawa, Y.; Kawamura, K. Characterization of bla(CTX-M27)/F1:A2:B20 plasmids harbored by Escherichia coli Sequence Type 131 Sublineage C1/H30R isolates spreading among elderly Japanese in nonacute-care settings. Antimicrob. Agents Chemother. 2020, 64, e00202-20. [CrossRef]

23. Zhang, W.H.; Lin, X.Y.; Xu, L.; Gu, X.X.; Yang, L.; Li, W.; Ren, S.Q.; Liu, Y.H.; Zeng, Z.L.; Jiang, H.X. CTX-M-27 producing Salmonella enterica Serotypes typhimurium and indiana are prevalent among food-producing animals in China. Front. Microbiol. 2016, 7, 436. [CrossRef] [PubMed]

24. Kawamura, K.; Sugawara, T.; Matsuo, N.; Hayashi, K.; Norizuki, C.; Tamai, K.; Kondo, T.; Arakawa, Y. Spread of CTX-type extended-spectrum $\beta$-lactamase-producing Escherichia coli isolates of epidemic clone B2-O25-ST131 among dogs and cats in Japan. Microb. Drug Resist. 2017, 23, 1059-1066. [CrossRef] [PubMed]

25. Piccirilli, A.; Pompilio, A.; Rossi, L.; Segatore, B.; Amicosante, G.; Rosatelli, G.; Perilli, M.; Di Bonaventura, G. Identification of CTX-M-15 and CTX-M-27 in antibiotic-resistant Gram-negative bacteria isolated from three rivers running in Central Italy. Microb. Drug Resist. 2019, 25, 1041-1049. [CrossRef] [PubMed]

26. Piccirilli, A.; Cherubini, S.; Azzini, A.M.; Tacconelli, E.; Lo Cascio, G.; Maccacaro, L.; Bazaj, A.; Naso, L.; Amicosante, G.; LTCFVeneto Working Group; et al. Whole-Genome Sequencing (WGS) of carbapenem resistant K. pneumoniae isolated in Long-Term Care Facilities in the Northern Italian Region. Microorganisms 2021, 9, 1985. [CrossRef] [PubMed] 